\title{
Histopathologic risk factors in oral and oropharyngeal squamous cell carcinoma variants: An update with special reference to HPV-related carcinomas
}

\author{
Samir K El-Mofty
}

DMD, MS, PhD. Professor. Washington University School of Medicine, Department of Pathology and Immunology

Correspondence:

Campus Box 8118

Washington University School of Medicine

Department of Pathology and Immunology

660 South Euclid Avenue, St Louis

MO. 63110, USA

elmofty@wustl.edu

Received: 20/05/2014 Accepted: $23 / 05 / 2014$

\author{
El-Mofty SK. Histopathologic risk factors in oral and oropharyngea \\ squamous cell carcinoma variants: An update with special reference to \\ HPV-related carcinomas. Med Oral Patol Oral Cir Bucal. 2014 Jul 1;19 \\ (4):e377-85. \\ http://www.medicinaoral.com/medoralfree01/v19i4/medoralv19i4p377.pdf \\ Article Number: $20184 \quad$ http://www.medicinaoral.com \\ (c) Medicina Oral S. L. C.I.F. B 96689336 - pISSN 1698-4447 - eISSN: $1698-6946$ \\ eMail: medicina@medicinaoral.com \\ Indexed in: \\ Science Citation Index Expanded \\ Journal Citation Reports \\ Index Medicus, MEDLINE, PubMed \\ Scopus, Embase and Emcare \\ Indice Médico Español
}

\begin{abstract}
Accurate identification of the microscopic risk factors of oral and oropharyngeal (OP) squamous cell carcinomas (SCC) and their morphologic variants is of at most importance, as these generally determine treatment modalities, prognosis and overall patient outcome. The great majority of oral and oropharyngeal squamous cell carcinomas are microscopically described as kerartinizing squamous cell carcinoma (KSCC). They bear certain resemblance to keratinizing stratified squamous epithelium. Tobacco habits and excessive consumption of alcoholic beverages have been considered to be the main etiologic agents in these carcinomas. The tumors occurred in older patients more commonly affected the oral tongue and floor of the mouth with well established morphologic risk factors including tumor grade, pattern of invasion and perineural involvement.

Within the last 30 years however, the advent and expanding prevalence of high risk human papillomavirus (HPV) as an important etiologic agent for head and neck squamous cell carcinoma, particularly in the OP, has resulted in a significant change in the established morphologic criteria for risk assessment. The majority of HPV relate carcinomas of the OP are nonkeratinizing squamous cell carcinoma (NKSCC). These tumors are found to be more responsive to treatment with a favorable patient outcome and good prognosis. Consequently, alterations in treatment protocols aimed at de-escalation are currently being evaluated. More recently, other morphologic variants that are HPV positive are reported with increasing frequency in the OP and other head and neck sites. As a result, several clinical and pathologic questions have emerged. Importantly, whether the virus is biologically active in these tumors and involved in their pathogenesis, and second, what are the clinical implications with regard to patient management and outcome in the HPV-related variants.

Examples of HPV-related squamous cell carcinoma variants that will be addressed here are: basaloid squamous cell carcinoma (BSCC), undifferentiated carcinoma (UCa), papillary squamous carcinoma (PSCC) and small cell carcinoma. Some studies have suggested favorable prognosis in some variants, analogous to that of the (NKSCC), while others showed poorer outcome. So far the number of studies on this subject is limited and the number of cases evaluated in each investigation is few. Because of that, it is prudent at this stage, not to alter management protocols as a result of identification of HPV in these variants and to await additional information.
\end{abstract}


Key words: Histopathologic risk-factors, oral cavity, oropharynx, squamous cell carcinoma variants, keratinizing squamous cell carcinoma, nonkeratinizing squamous cell carcinoma, HPV, basaloid squamous cell carcinoma, undifferentiated carcinoma, papillary squamous cell carcinoma, small cell carcinoma.

\section{Keratinizing squamous cell carcinoma (KSCC); morphologic risk factors}

Traditionally KSCCs have been graded according to their state of differentiation and resemblance to normal squamous epithelium into well, moderate and poorly differentiated variants. The grading system which was originally proposed by Broder (1) was based on the amount of keratin production and pleomorphism of the tumor cells. However, because the morphologic features can vary considerably from area to area within the same tumor, the Broder grading system was found to lack significant prognostic value. Several authors suggested that more useful prognostic information may be deduced from the invasive fronts of the tumors where the deepest and presumably more aggressive cells reside $(2,3)$. According to this system five histologic features are graded and assigned scores from one to four. The scores for all the variant are summed to provide a total malignancy score for a particular tumor. The parameters used are: degree of keratinization, nuclear pleomorphism, number of mitosis per high power field (HPF), pattern of invasion and inflammatory lymphoplasmacytic host response. The highest scores are given to tumors with low or no keratinization, extreme nuclear pleomorphism, $>5$ mitosis/HPF, marked cellular dissociation in small nests and single cells and no host response.

A newer risk model was developed by BrandweinGensler and associates (4-6) as an extension and modification of prior multivariable histologic systems analyzing the advancing tumor front. In this model a risk category is assigned by examining the resection specimen of the primary tumor and quantifying 3 significant histologic variables; 1- Pattern of invasion at the advancing tumor edge (PI). The different types of patterns are described as; pushing border, finger like growth, large islands, small islands or distant satellites. 2- Perineural invasion (PNI) involving either small nerves or large ones (> 1mm). 3- Lymphocytic host response at the advancing tumor edge described as strong, intermediate or weak. The risk model classified the tumors, according to a point score into low, intermediate, and high risk groups which correlated significantly with local recurrence rates and overall survival in a cohort of patients with head and neck squamous cell carcinoma (5). The prognostic value of this risk model was further validated in patients with low-stage (Stag I/II) oral squamous cell carcinoma. The model was significantly predictive of loco-regional recurrence and disease specific sur- vival (6). Based on these observations it was suggested that some low stage oral SCC that would traditionally be treated surgically may benefit from adjuvant radiotherapy if classified in the high risk group. Tumor thickness in oral KSCC has also been used as a parameter for outcome prediction in T1 carcinomas. However, it was found that the cut-off tumor thickness varied by anatomic site. In addition, it predicted lymph node metastasis and survival but not local recurrence.

\section{HPV-Related squamous cell carcinoma}

During the last 30 years accumulating epidemiologic and clinical evidence has shown that high risk human papillomavirus (HPV) is a major etiologic factor in a subset of head and neck squamous cell carcinomas. These tumors have distinct clinical, microscopic and molecular features. The greatest majority of the carcinomas occur in the OP, particularly the palatine and lingual tonsils areas. They are characterized by younger age at onset (7), weak or no association with alcohol and tobacco use but strong association with sexual behavior, particularly oral sex $(8,9)$. Despite a characteristic early lymph node metastasis, HPV-related oropharyngeal squamous cell carcinomas are associated with significantly better treatment outcome and patient survival (10-12).

Microscopically, HPV-related squamous cell carcinoma of the OP are distinguished by a nonkeratinizing morphology $(7,11,13)$. The tumors are characterized by relatively monomorphic, ovoid and spindle-shaped basaloid cells with indistinct cell border (Fig. 1). The nuclei are hyperchromatic with high nuclear-to-cytoplasmic ratio. The cells form sheets, nests and cords with sharply defined borders. Excessive mitosis and apoptosis as well as comedo-type necrosis are observed. These carcinomas also show a distinct immunohistochemical profile namely, a strong and diffuse p16 reactivity, very high Ki-67 labeling scores (Fig. 1) and negative or week staining with p53. A hybrid form of NKSCC in which the basaloid tumor cells show focal and partial keratinocytic maturation has been described by El-Mofty et $a l$, (13) (Fig. 2). This hybrid variant was shown to be HPV related and to also have a favorable clinical outcome (11).

p16 over expression is now considered a surrogate marker for HPV-related NKSCC of the OP $(7,14)$. p16 is a cell cycle protein associated with tumor suppression by the retinoblastoma pathway. It inhibits hyperphosphorilation of retinoblastoma protein $(\mathrm{pRb})$ thus preventing its dissociation from the transcription factor E2F and the subse- 


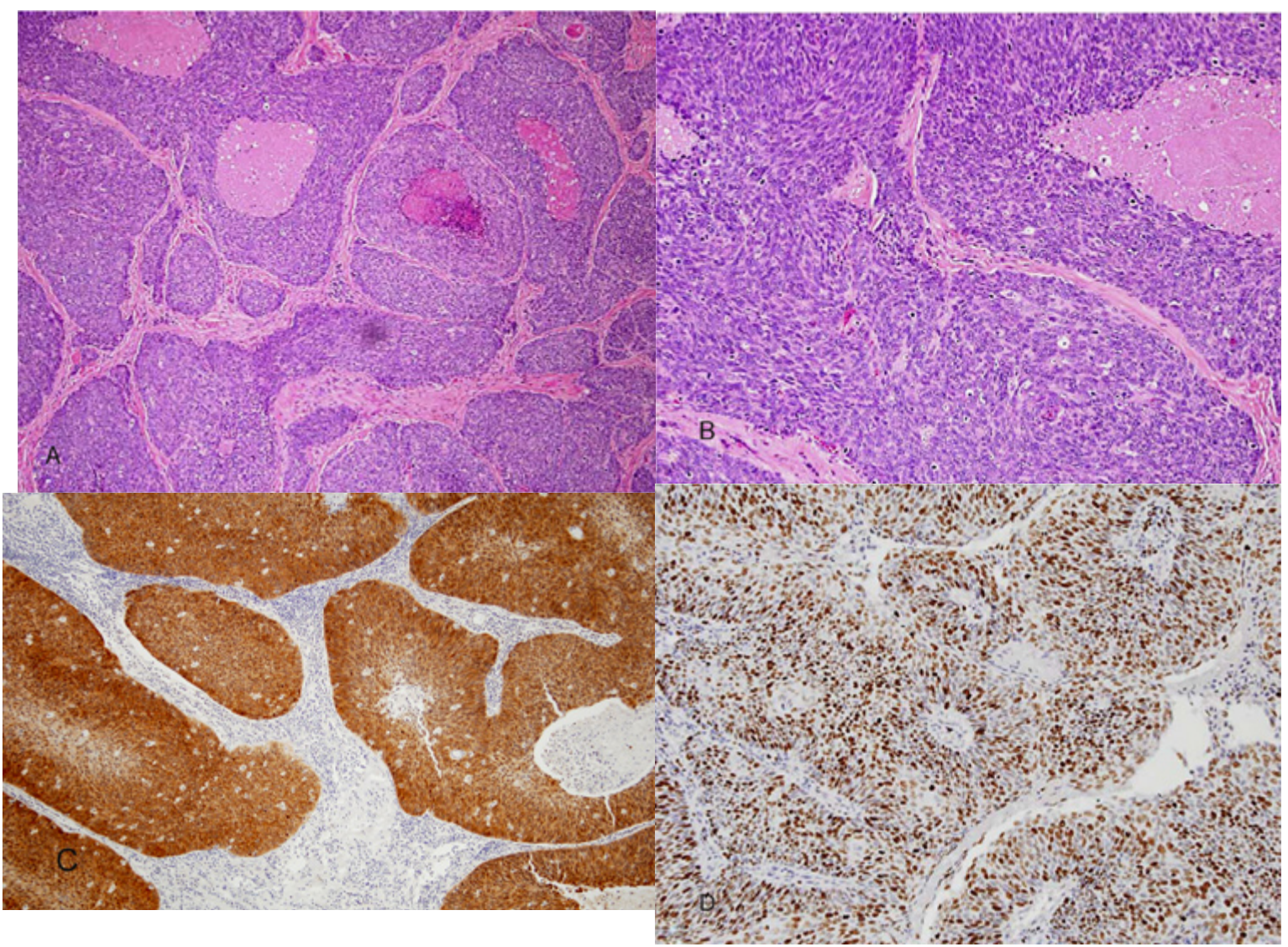

Fig. 1. A) Nonkeratinizing squamous cell carcinoma. Sheets and large nests of tumor cells are seen with well defined borders and central comedo necrosis. B) Higher magnification showing small ovoid and spindle shaped tumor cells with hyperchromatic nuclei and indistinct cell bordrs. C) Strong and diffuse p16 immunostaining, both nuclear and cytoplasmic. D) Ki-67stain showing high labeling score of more than $80 \%$.
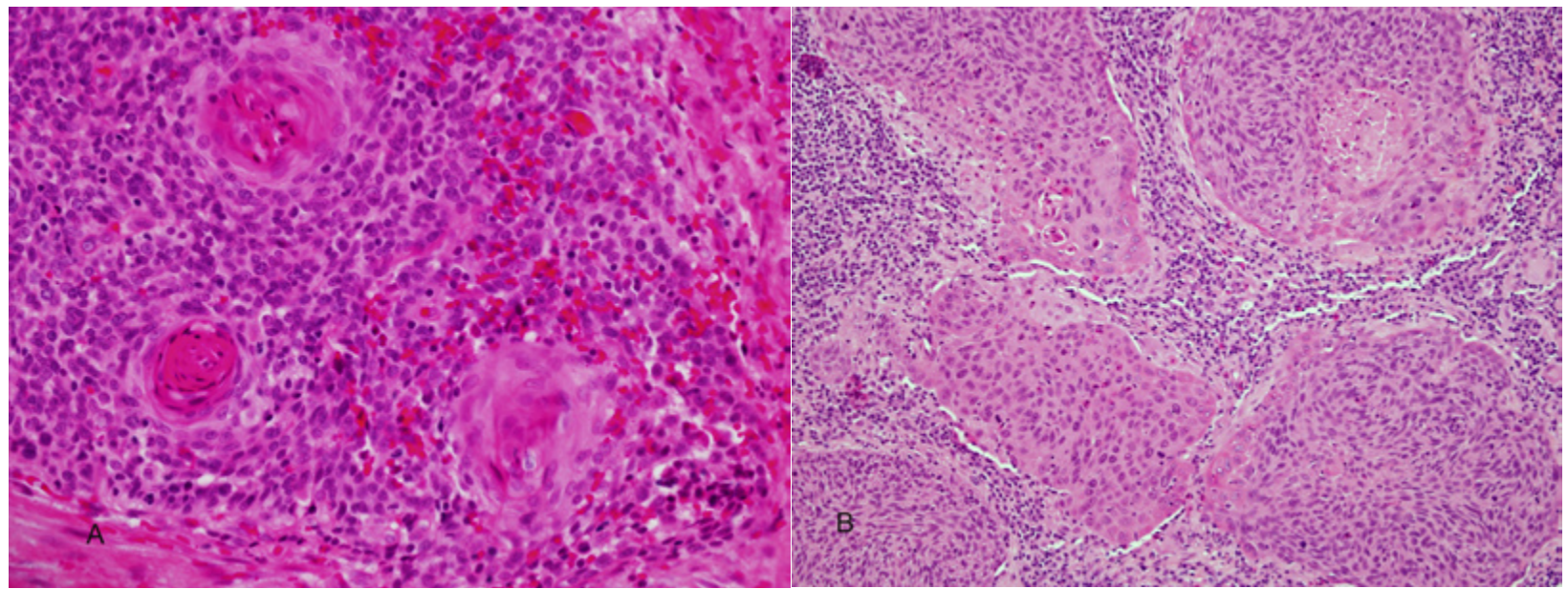

Fig. 2. A) Nonkeratinizing squamous cell carcinoma with focal areas of keratinocytic maturation (hybrid variant). B) Nonkeratinizing hybrid variant with peripheral partial maturation of basaloid cells.

quent progression of the $\mathrm{S}$ phase of the cell cycle $(15,16)$. HPV E7 oncoprotein interacts with $\mathrm{pRb}$ active form resulting in its functional inactivation. The paradoxical overexpression of an inhibitory protein in actively replicating neoplastic cells is thought to result from feed-back control secondary to $\mathrm{pRb}$ deregulation $(17,18)$.
It has been shown that accurately identifying the nonkeratinizing morphologic features and its hybrid variant in oropharyngeal squamous cell carcinomas can be used as predictor of favorable clinical outcome (19). The nonkeratinizing morphology is significantly more likely to be HPV and p16 positive than KSCC, and to have bet- 
ter overall survival (OS) and disease specific survival (DSS) with a $p$ value of $<0.001$ and 0.01 respectively (19). It was also shown that p16 overexpression in oropharyngeal SCC is associated with HPV positivity and has significantly better patient outcome (20). The pattern of p16 immunohistochemical staining that correlates with transcriptionally active HPV and better patient outcome is strong and diffuse both nuclear and cytoplasmic in more than $75 \%$ of the tumor cells (Fig. 1) $(20,21)$.

Because of the observed disparity in clinical outcome of squamous cell carcinomas consequent to their HPV status, there are ongoing clinical investigations to test the efficacy of altering treatment paradigms in documented HPV- related tumors. Multi-institutional clinical trials are underway to determine the feasibility of de-escalated treatment modalities in these cases. It is therefore of importance that HPV status should be reliably and accurately determined. Detection p16 overexpression by immunohistochemistry is the most commonly used technique for this purpose. However in rare occasions p16 is not specific. Another common detection method is in situ hybridization (ISH) for HPV DNA. While highly specific it is not very sensitive. On the other hand, PCR analysis for HPV DNA is highly sensitive but not necessarily specific. Detecting viral DNA by PCR does not indicate whether the virus is trancriptionally active (driver) or a bystander (passenger).

E6/E7 mRNA expression is considered the "gold standard" for identification of clinically significant HPV infection in tumor specimens. Reverse transcriptase polymerase chain reaction (RT-PCR) and real-time quantitative RT-PCR (RT-qPCR) are the main methods used for detection and quantitation of E6/E7 mRNA $(22,23)$. More recently, an in situ hybridization method has been developed for detection of transcriptionally-active HPV in head and neck squamous cell carcinomas. ISH for E6/E7 mRNA is a slide-based chromogenic assay that has been developed under the name RNA scope (Advanced cell diagnostics, Inc., Hayward, CA) $(24,25)$. Results from HPV E6/E7 mRNA ISH were found to be highly concordant with p16 immunohistochemistry and RT-qPCR $(24,25)$. A small minority of HPV+ OP SCC show atypical clinical behavior following treatment including poor OS, DSS and Disease free survival (DFS) $(12,26,27)$. Some cases may be associated with multicentric disease in the mucosa of the upper aerodigestive tract excluding the oral cavity. The reported sites of involvement include the larynx, nasopharynx and sinonasal tract (28). Also, on rare occasion HPV+ OP SCC was reported to have multiple distant metastasis to unusual sites including the skin brain, gastrointestinal tract and intra-abdominal lymph nodes $(28,29)$ The interval from completion of therapy to onset of distant metastasis ranged from 4- 11 (28) and 2-52 (29) months with a median of onset 7 and 18 months respectively $(28,29)$.
The exact causal mechanisms involved in poor clinical outcome and patient survival in a minority of HPV positive SCC of the oropharynx are not clear. However, certain relevant molecular profiles have been proposed. These include over-expression of Bcl2, EGFR, and BclxL, $(27,30,31)$. EGFR is a transmembrane tyrosine kinase, its function affects cell cycle progression, apoptosis, angiogenesis, tumor cell mobility and metastasis. Over expression of EGFR was shown to be associated with poor OS and DFS in HPV +, p16 + SCC of the oropharynx $(30,31)$. Similarly over-expression of the antiapoptotic proteins Bcl2 and BclxL was shown to be associated with poor OS and DFS as well as DSS in $\mathrm{HPV}+\operatorname{OPSCC}(27,31)$ these proteins are known to confer resistance to chemotherapy and radiation therapy of head and neck SCC. It has also been documented that patients who continue to smoke after treatment have a significantly worse DSS than those who were past or never smokers $(12,31)$.

\section{HPV Related squamous cell carcinoma of the oral cavity}

Unlike the OP the oral cavity proper is a rare site for HPV related SCC. El-Mofty and LU (7), using PCR and p16 immunostain, have shown in a cohort of young patients who are 40 years of age or younger that the prevalence of HPV related SCC of the palatine tonsils was $(91 \%)$ 10/11 while in the oral cavity the prevalence was $(0 \%) 0 / 15$. However, considerable variations in the prevalence of HPV in oral SCC have been reported in the literature. These variations may be attributable to differences in specificity and sensitivity of the tests used and whether the identified virus is a "driver" or a "passenger" in these tumors. Prevalence may also vary by geographic distribution of the studied cases. A range of between $0 \%-11 \%$ has been reported $(7,32-35)$. HPV+ oral SCC, unlike those in the OP, do not exhibit nonkeratinizing morphology. The significance of HPV in oral SCC and its relationship to treatment outcome and patient's survival is currently not known.

\section{HPV-Related SCC variants}

The great majority of HPV relate SCC of the OP is nonkeratinizing as described above. However, more recently increasing numbers of variants of squamous cell carcinoma, that are HPV-positive, are reported in the oropharynx as well as in other head and neck sites. As a result, several clinical and pathologic questions have emerged. Importantly, whether the virus is biologically active and involved in the pathogenesis of these tumors, and whether there are clinical implications with regard to patient outcome and treatment modality changes that may be needed in HPV related variants. Examples of HPV-related squamous cell carcinoma variants that will be addressed here include: basaloid squamous cell 
carcinoma, undifferentiated carcinoma, papillary squamous carcinoma, small cell carcinoma and keratinizing squamous cell carcinoma. Some investigations have suggested favorable prognosis in some HPV positive variants, analogous to that of the nonkeratinizing carcinoma, while others showed poorer outcome. So far the number of studies on this subject is limited and the number of cases evaluated in each investigation is few. Because of this, it is prudent that at this stage, not to alter management protocols as a result of identification of HPV in these variants and to await additional studies.

\section{Basaloid squamous carcinoma (BSCC)}

In the upper aerodigestive tract, $\mathrm{BSCC}$ is rare variant of conventional SCC. It occurs more commonly in the hypopharynx and larynx and less frequently in the oropharynx. Like SCC the tumor is typically associated with traditional risk factors like tobacco smoking and alcohol abuse. It is generally considered a high grade malignant neoplasm with poor prognosis. Microscopically, it is characterized by a biphasic pattern. A basaloid component intimately associated with elements of keratinizing SCC. The basaloid cells are small, crowded with hyperchromatic round nuclei and scant cytoplasm. They form sheets and lobules that produce a "jigsaw puzzle" growth pattern with cystic spaces containing PAS - positive myxoid material. Stromal hyalization may be present (Fig. 3). The squamous component is either surface SCC or severe dysplasia in addition to focal abrupt squamous differentiation within the basal cell areas (Fig. 3). These microscopic features are distinct from but may be confused with those of NK SCC as described above.

A causal relationship between HPV and some cases BSCC has been documented in the upper aerodigestive tract. Chernock et al, (36) using ISH for HPV DNA and p16 immunohistochemistry found that $75 \%$ of orophryngeal BSCC are HPV related while none $(0 \%)$ of 16 laryngeal/hypopharyngeal tumors were positive (36). Using PCR for HPV 16 DNA as well as p16 immunostaining, Begum and Westra (37) documented HPV in $76 \%$ of oropharyngeal BSCC and in $6 \%$ of tumors in non-oropharyngeal sites. In both studies $(36,37)$ the overall survival was significantly better in HPV positive tumors than in the HPV negative ones with $p<0.05$ and $<0.0001$ respectively.

\section{Undifferentiated carcinoma (lymphoepithelial, nasopharyngeal type)}

In the head and neck undifferentiated carcinoma (UDCa) is best recognized in the nasopharynx where it is referred to by a variety of names including; lymphoepithelioma, nasopharyngeal type undifferentiated carcinoma and nonkeratinizing undifferentiated carcinoma (WHO type III). Microscopically identical carcinomas are also identified in the oropharynx. While undifferentiated carcinoma of the nasopharynx has a strong etiologic relationship to Epstein-Barr virus

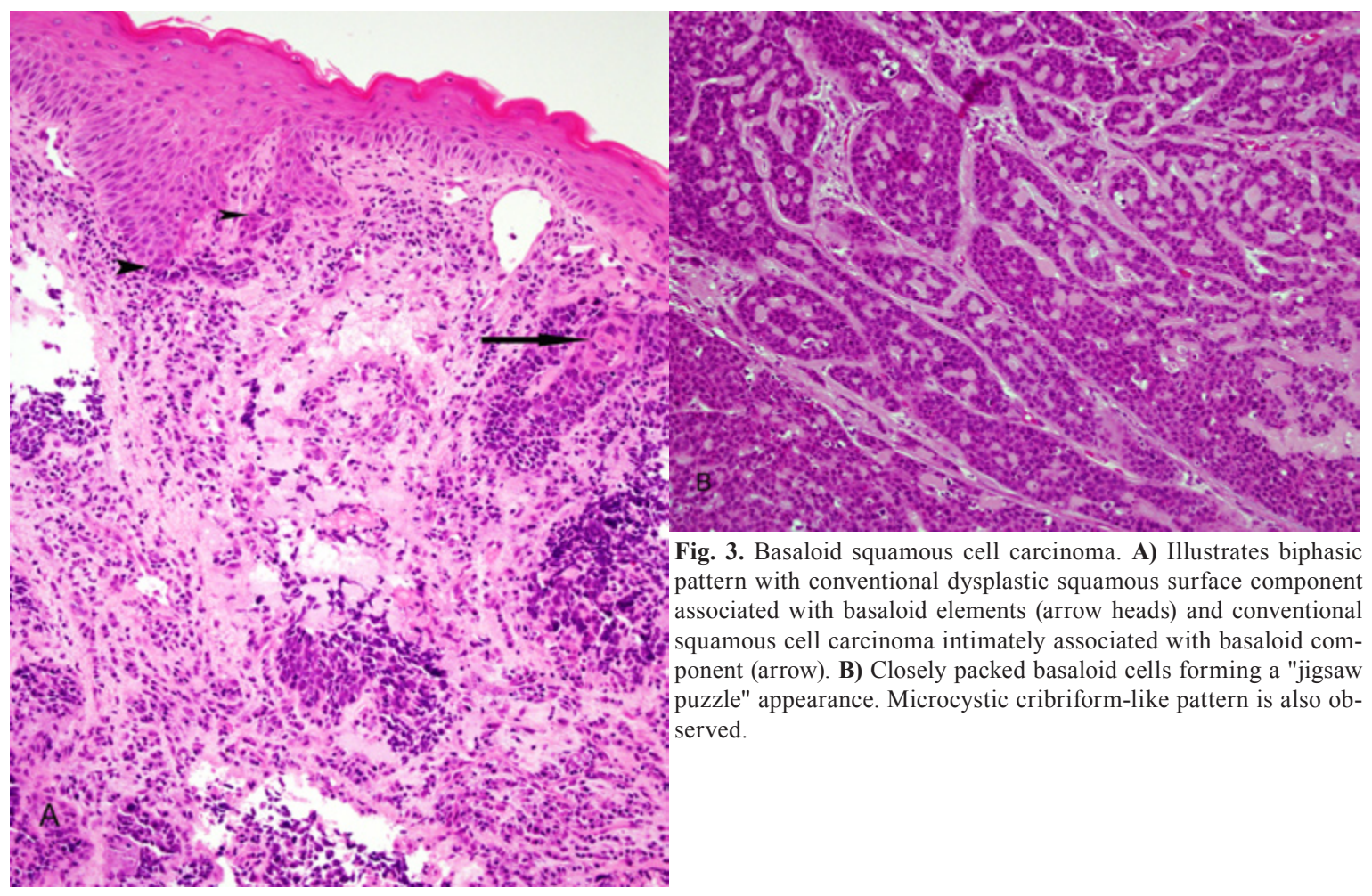


(EBV) (38), undifferentiated carcinoma of the oropharynx has recently been shown to be predominantly HPV and not EBV-related $(39,40)$.

The microscopic features of oropharyngeal undifferentiated carcinoma are indistinguishable from those of the nasopharyngeal type as defined by the WHO. The tumors are composed of solid sheets, trabeculae, nests and single neoplastic epithelial cells intimately intermingled with lymphocytes and plasma cells. The epithelial tumor cells are large with indistinct cell borders forming a syncytium (Fig. 4). The nuclei are round to oval and vesicular with large central nucleoli. HPV DNA was identified in these tumors by PCR and ISH. Evidence for biologic activity of the virus was demonstrated by p16 over- expression $(39,40)$. The 3 year OS was found to be $55 \%$, while the DSS was $100 \%$ (39). No tumor recurrence was observed during a median follow up period of 23 months (40). The patient's outcome in HPV positive undifferentiated carcinoma of the OP is generally favorable and comparable to that of the nonkeratinizing HPV-related squamous cell carcinoma.

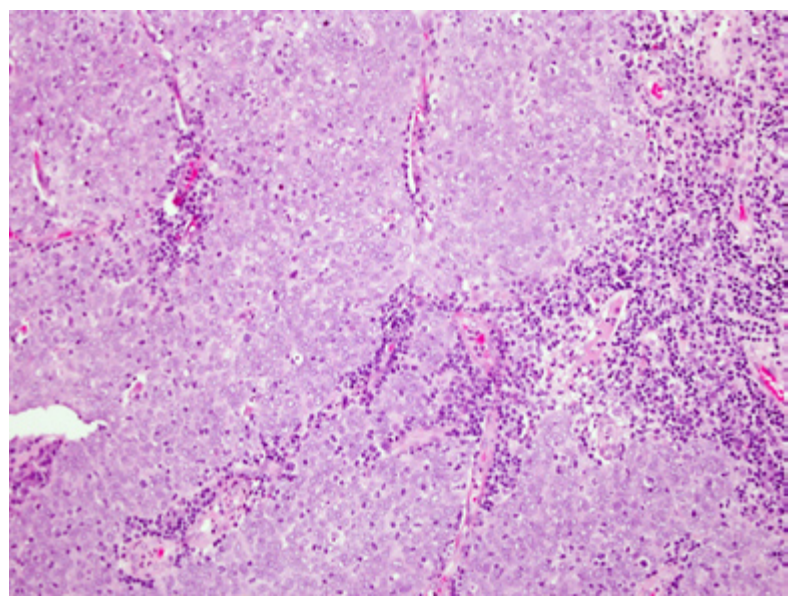

Fig. 4. Undifferentiated (lymphoepithelial) carcinoma. Undifferentiated epithelial cells forming a syncytium and intermingled with lymphocytes and plasma cells. The tumor cells have large vesicular nuclei.

\section{Papillary squamous cell carcinoma}

Papillary squamous cell carcinoma (PSCC) is a poorly understood variant of SCC of the upper aerodigestive tract that is often confused with other exophytic mucosal malignancies such as verrucous carcinoma and squamous cell carcinoma with verrucous features. As defined by the WHO, PSCC is characterized by a predominant papillary growth pattern with thin fibrovascular cores covered by immature basaloid cells or dysplastic cells with minimal or no keratinization. PSSC is generally believed to have a better prognosis than conventional squamous cell carcinoma.

Carcinomas with papillary growth pattern occurring in the uterine cervix, vulva and penis are commonly associated with High risk HPV particularly type 16. Very limited number of studies has so far investigated the prevalence and significance of HPV in PSCC of the head and neck $(41,42)$. Jo et al. (41) found that 15 of 31 cases of PSCC of the head and neck were both p16 and HPV ISH positive. The majority of those (11 cases) were oropharyngeal. Patient outcome in HPV positive and negative tumors was not adequately investigated.

In a recent study (42) we reviewed 48 cases of PSCC of the head and neck, 7 in the oral cavity 19 oropharynx and 22 in the larynx. Two morphologic types were identified: (1) a keratinizing type (K) in which the dysplastic epithelium showed maturation trend with minimal surface parakeratin, and a nonkeratinizing (NK) type in which the papillae were covered with immature basaloid cells (Fig. 5). An HPV relationship was identified by p16 immunoreactivity, HPV ISH and E6 and E7 mRNA ISH in a number of tumors (Fig. 5). The majorities of these were found in younger patients, occurred more commonly in the OP, had NK morphology and were less likely to be p53 positive. Disease specific survival (DSS) was favorable in all cases. The 5 years DSS for p16 positive and p16 negative cases was $80 \%$ and 70 $\%$ respectively. No statistically significant difference in OS, DSS or disease-free survival (DFS) was found regarding tumor site, morphologic type or HPV relationship. However, a trend towards better DFS was seen in patients with p16 positive/HPV positive tumors (42).

\section{Small cell (neuroendocrine) carcinoma}

An association between HPV and neuroendocrine carcinoma of the oropharynx was shown in two recent studies $(43,44)$. Bishop and Westra (43) found that 5 of 9 cases of oropharyngeal small cell carcinoma (poorly differentiated neuroendocrine carcinoma) were HPVrelated. In 4 of these cases the tumors were associated with typical HPV-related SCC. All 5 tumors were p16 positive by immunohostochemistry and HPV positive by ISH. All cases showed a characteristic neuroendocrine immunophenotype, including reactivity to synaptophysin and /or chromogranin and CAM 5.2 and were negative for CK 5/6. Three of the 5 patients died within 15 month of diagnosis (mean 10 months) with widely disseminated disease. Such outcome is in sharp contrast to the typical HPV-related nonkeratinizing SCC which is associated with a 3-year survival rate of up to $80 \%$ (12). Kraft et al. (44) reported 8 oropharyngeal neuroendocrine carcinomas that were HPV-related. Disease recurrence occurred in 5 of 6 patients with available clinical follow-up, with 3 developing metastasis to bone, lung, pleura, adrenal gland and pancreas.

HPV-related small cell carcinoma of the oropharynx shares common features with small cell neuroendocrine carcinoma of the uterine cervix. Both are associated with high risk HPV, commonly coexist with non-small 


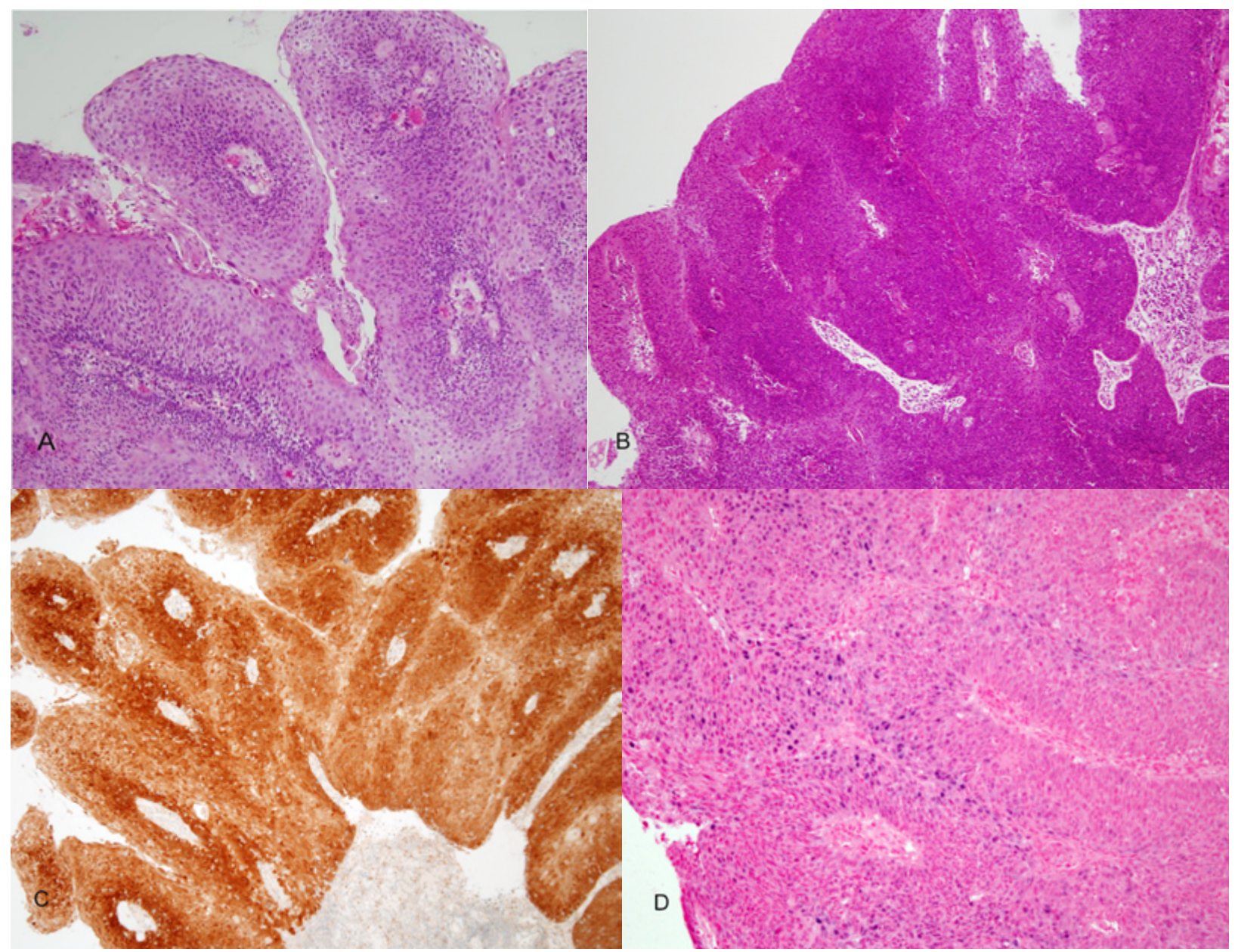

Fig. 5. Papillary squamous cell carcinoma. A) Keratinizing type, the dysplastic cells show maturation with minimal parakeratin formation. B) Nonkeratinizing type with immature basaloid cells. C) strong and diffuse p16 staining. D) Positive ISH for high risk-HPV DNA (blue nuclear staining).

cell squamous cell carcinoma and share the same aggressive clinical behavior with early distant metastasis and poor overall survival (43-46).

\section{HPV-Related keratinizing squamous cell carci- noma of the oropharynx}

Classical KSCC of the OP is becoming much less frequently encountered in some parts of the world including the United States and some European countries. A very small minority of these are HPV related. In a recent study we have found that only 7 of 54 (13\%) KSCC of the OP overexpressed p16. HPV E6/E7 RNA ISH was positive in 5 tested cases. The OS and DSS was significantly better in the p16 positive than the 16 negative KSCC cases ( $p=0.01$ and 0.04 ) respectively (47).

\section{Discussion}

The histopathologic features of keratinizing SCC of the upper aerodigestive tract have been used by Pathologists as tools for prediction of tumor behavior and patient prognosis. Consequently, Clinicians have relied on this information for determining treatment modalities to be used in each particular case. The greatest majority of these keratinizing neoplasms shared morphologic risk features that are used to classify them in a range of grades, from low to high. Less frequently, variant forms of KSCC such as BSCC, PSCC, adenosquamous carcinoma, spindle cell carcinoma and UCa are encountered. These variants have distinct morphology and clinical behavior and are not graded.

Within the last few decades an HPV related nonkeratinizing variant of SCC was identified. The majority of which occurred in the oropharynx. The neoplastic cells in these tumors show an immature basaloid appearance and are mitotically active. Such phenotype is not limited to oropharyngeal sites but it is also observed in other HPV related tumors in variety of sites including the sinonasal tract, larynx the and anogenital sites $(48,49)$. The exact molecular mechanisms underlying the expression of this specific histopathologic phenotype are not clear. It is possible that interactions between HPV oncoproteins and cell cycle mediators may play a role. It 
is known that high risk HPV oncoproteins, particularly $\mathrm{E} 6$ and $\mathrm{E} 7$, interfere with $\mathrm{Rb}$ and $\mathrm{p} 53$ functions leading to cell cycle progression, cell immortalization, suppression of apoptosis and essentially uncoupling of proliferation and maturation. It is conceivable that these changes may be responsible for the characteristic immature basaloid appearance of the tumor cells. Chromosomal instability is another property of HPV oncogenesis. It is likely that additional mutations may be responsible for the development of those rare microscopic SCC variants discussed above.

As the numbers of reported HPV positive head and neck squamous cell carcinoma variants are increasing, particularly in the oropharynx, it is of importance to establish the status of the virus in the tumors cells and to distinguish between a causal agent "driver" and a bystander "passenger". Currently, the number of cases of SCC variants with established HPV causal relationship is still limited. Based on these studies, there is some evidence to suggest that variants such as $\mathrm{BSCC}$, undifferentiated carcinoma and PSCC may have favorable prognosis similar to that of conventional NKSCC and better than their HPV negative counterparts. On the other hand, HPV related small cell carcinoma has been shown to be associated with early distant metastasis and poor overall survival. More over a minority of NKSCC of the oropharynx have been recently found to be associated with atypical clinical behavior following treatment, including poor OS, DSS and DFS $(12,26,27)$.It is thus too early to reach a definite conclusion regarding the biologic behavior of HPV-related SCC morphologic variants. Surgeons and oncologists should be warned that HPV status in these tumors should not be used, at this time, as a justification for considering deescalation of conventional therapy.

\section{References}

1. Broder AC. The grading of carcinoma. Minn Med. 1925;8:726. 2. Bryne M, Koppang HS, Lilleng R, Stene T, Bang G, Dabelsteen E. New malignancy grading is a better prognostic indicator than Broders' grading in oral squamous cell carcinomas. J Oral Pathol Med. 1989;18:432-7.

3. Bryne M, Koppang HS, Lilleng R, Kjaerheim A. Malignancy grading of the deep invasive margins of oral squamous cell carcinomas has high prognostic value. J Pathol. 1992;166:375-81.

4. Brandwein-Gensler M, Teixeira MS, Lewis CM. Oral squamous cell carcinoma: histologic risk assessment, but not margin status, is strongly predictive of local disease-free and overall survival. Am J Surg Pathol. 2005;29:167-178.

5. Brandwein-Gensler M, Smith RV, Wang B, Penner C, Theilken A, Broughel D, et al. Validation of the histologic risk model in a new cohort of patients with head and neck squamous cell carcinoma. Am J Surg Pathol. 2010;34:676-688.

6. Li Y, Bai S, Carroll W. Validation of the risk model: High risk classification and tumor pattern of invasion predict outcome for patients with low stage oral cavity squamous cell carcinoma. Head Neck Pathol. 2013;7:211-223.

7. El-Mofty SK, Lu DW. Prevalence of human papillomavirus type 16 DNA in squamous cell carcinoma of the palatine tonsil, and not the oral cavity, in young patients: a distinct clinicopathologic and molecular disease entity. Am J Surg Pathol. 2003;27:1463-70.
8. Chaturvedi AK. Epidemiologic and clinical aspects of HPV in head and neck cancer. Head Neck Pathol. 2012;6:S16-S24.

9. Gillison ML, D'Souza G, Westra W, Sugar E, Xiao W, Begum S, et al. Distinct risk factor profiles for human papillomavirus type 16positive and human papillomavirus type-16 negative head and neck cancer. J Natl Cancer Inst. 2008;100:407-420.

10. Fakhry C, Westra WH, Li S, Cmelak A, Ridge JA, Forestiere A, et al. Improved survival of patients with human papillomavirus positive head and neck squamous cell carcinoma in a prospective clinical trial. J Natl Cancer Inst. 2008;100:261-9.

11. Chernock RD, El-Mofty SK, Thorstad WL, Parvin CA, Lewis JS. HPV-related nonkeratinizing squamous cell carcinoma of the oropharynx: utility of microscopic features in predicting patient outcome. Head neck Pathol. 2009;3:186-194.

12. Ang KK, Harris J, Wheeler R, Weber R, Rosenthal DI, NguyenTan PF, et al. Human papillomavirus and survival of patients with oropharyngeal cancer. N Eng J Med. 2010;363:24-35.

13. El-Mofty SK, Zhang MQ, Davila RM. Histologic identification of human Papillomavirus (HPV)-related squamous cell carcinoma in cervical lymph nodes: a reliable predictor of the site of an occult head and neck primary carcinoma. Head Neck Pathol. 2008;2:163-8.

14. El-Mofty SK, Patil S. Human papillomavirus (HPV)-related oropharyngeal nonkeratinizing squamous cell carcinoma: characterization of a distinct phenotype. Oral Surg Oral Med Oral Pathol Oral Radiol Endod. 2006;101:339-45.

15. Syrjanen SM, Syrjanien KJ, Lobe DC. New concepts on the role of human papillomavirus in cell cycle regulation. Ann Med. 1999;31:175-187.

16. Phelps WC, Barnes JA, Lobe DC. Molecular targets of human papillomavirus: prospects for antiviral therapy. Antivir Chem chemother. 1998;9:359-377.

17. Wilczynski SP, Lin BT, Xie Y, Paz IB. Detection of human papillomavirue DNA and protein overexpressiomn are associated with a distinctmorphological pattern of tonsillar squamous cell carcinoma. Am J Pathol. 1998:152:145-156.

18. Klaes R, Friedrich T, Spitkovsky D, Ridder R, Rudi W, Petry U, et al. Overexpression of p16(INK4A) as a specific marker for dysplastic and neoplastic cells of the cervix uteri. Int J Cancer. 2001;92:276284.

19. Chernock RD, El-Mofty SK, Thorstad WL, Parvin CA, Lewis JS. HPV-related nonkeratinizing squamous cell carcinoma of the oropharynx: Utility of microscopic features in predicting patient outcome. Head Neck pathol. 2009;3:186-194.

20. Lewis JS, Thorstad WL, Chernock RD, Haughey BH, Yip JH, Zhang Q, et al. p16 positive oropharyngeal squamous cell carcinoma: an Entity with favorable prognosis regardless of tumor HPV status. Am J Surg Pathol. 2010;34:1088-1096.

21. Lewis JS, Chernock RD, Ma X-J, Flanagan JJ, Yuling L, Gao G, et al. Partial p16 staining in oropharyngeal squamous cell carcinoma: extent and pattern correlate with human papillomavirus RNA status. Mod Pathol. 2012;25:1212-1220.

22. Molden T, Krause I, Skomedal H, Nordstrom T, Karlsen F. PreTech HPV-proofer; real time detection and typing of E6/E7 mRNA from carcinogenic human papillomavirus. J Viro Methods. 2007;142:204-212.

23. Gao G, Chernock RD, Gay HA, Thorstad WL, Zhang TR, Wang $\mathrm{H}$, et al. A novel RT-PCR method for quantification of human papilomavirus transcripts in archival tissue and its application in oropharyngeal cancer prognosis. Int J Cancer. 2013;132:882-890.

24. Bishop JA, Ma XJ, Wang H, Luo Y, Illei BB, Begun S, et al. detection of transcriptionally active high risk HPV in patients with head and neck squamous cell carcinoma as visualized by a novel E6/E7 mRNA in situ hybridization method. Am J Surg Pathol. 2012;36:1874-1882.

25. Ukpo OC, Flanagan JJ, Ma XJ, Luo Y, Thorstad WL, Lewis JS. High-risk human papillomavirus E6/E7 mRNA detection by a novel in situ hybridization assay strongly correlates with p16 expression and patient outcomes in oropharyngeal squamous cell carcinoma. Am J Surg Pathol. 2011;35:1343-1350. 
26. Lewis JS. Scantlebury JB, Luo J, Thorstad WL. Tumor cell anaplasia and multinucleation are predictor of disease recurrence in oropharyngeal squamous cell carcinoma, including among just the Human papillomavirus-related cancers. Am J Surg Pathol. 2012;36:1036-1046.

27. Nicholas AC, Finkelstein DM, Faquin WC, Westra WH, Mroz $\mathrm{EA}$, Kneuertz $\mathrm{P}$, et al. Bcl2 and human papillomavirus 16 as predictor of outcome following concurrent chemoradiation for advanced oropharyngeal cancer. Clin Cancer Res. 2010;16:2138-2146.

28. Huang SH, Perez-Ordonez B, Liu FF, Waldron J, Ringash J, Irish $\mathrm{J}$, et al. Atypical clinical behavior of p16 confirmed HPV related oropharyngeal squamous cell carcinoma treated with radical radiotherapy. Int J Radiation Oncology Biol Phys. 2012;82:276-283.

29. Muller S, Khuri F, Kono SA, Beitler JJ, Shin DM, Saba NF. HPV positive squamous cell carcinoma of the oropharynx. Are we observing an unusual pattern of metastasis. Head Neck Pathol. 2012;6:336344.

30. Reimers N, Kasper HU, Weissenborn SJ, Stutzer H, Preuss FS, Hoffmann TK, et al. Combined analysis of HPV-DNA and EGFR expression to predict prognosis in oropharyngeal cancer. Int $\mathrm{J}$ Cancer. 2007;120:1731-8.

31. Kumar B, Gordell KG, Lee JS, Worden FP, Prince ME, Tran HH, et al. EGFR, p16, HPV titer, Bcl-xL and p53, sex and smoking as indicators of response to therapy and survival in oropharyngeal cancer. J Clin Oncol. 2008;26:3128-3137.

32. Ha PK, Pai SI, Westran WH, Gillison ML, Tong BC, Sidransky $\mathrm{D}$, et al. Real time quantitative PCR demonstrates low prevalence of human papillomavirus type 16 in premalignant and malignant lesions of the oral cavity Clin Cancer Res. 2002;8:1203-9.

33. Braakhuis BJ, Snijders PJ, Keune WJ, Meijer CJ, Ruijter-Schippers HJ, Leemans CR, et al. Genetic patterns in head and neck cancers that contain or lack transcriptionally active human papillomavirus. J Natl Cancer Inst. 2004;96:998-1006.

34. Schlecht NF, Brandwein-Gensler M, Nuovo GJ, Li M, Dunn A, Kawachi N, et al. A comparison of clinically utilized papillomavirus detection methods in head and neck cancer. Mod pathol. 2011;24:1295-1305.

35. Lewis JS, Ukbo OC, Ma X-J, Flanagan JJ, Luo Y, Thorstad WL, et al. Transcriptionally-active high-risk human papillomavirus is rare in oral and laryngeal/hypopharyngeal squamous cell carcinoma- a tissue microarray study utilizing E6/E7 mRNA in situ hybridization. Histopathology. 2012;60:982-991.

36. Chernock RD, Lewis JS, Zhang Q, El-Mofty SK. Human papillomavirus positive basaloid squamous cell carcinoma of the upper aerodigestive tract: a distinct clinicopathologic and molecular subtype of basaloid squamous cell carcinoma. Hum Pathol. 2010;41:1016-1023. 37. Begum S, Westra WH. Basaloid squamous cell carcinoma of the head and neck is a mixed variant that can be further resolved by HPV status. Am J Surg Pathol. 2008;32;1044-1050.

38. Nicholls JM, Agathanggelou A, Fung K, Zeng X, Niedobitek G. The association of squamous cell carcinomas of the nasopharynx with Epstein-Barr virus shows geographical variation reminiscent of Burkitt's lymphoma. J Pathol. 1997;183:164-8.

39. Carpenter D, El-Mofty SK, Lewis JS. Undifferentiated carcinoma of the oropharynx: A human papillomavirus associated tumor with favorable prognosis. Mod Pathol. 2011;24:1306-1312.

40. Singhi AD, Stelow EB, Mills SE, Westra WH. Lymphoepitheliallike carcinoma of the oropharynx ; A morphologic variant of HPV related head and neck carcinoma. Am J Surg Pathol. 2010;34:800-805. 41. Jo VY, Mills SE, Stoler MH, Stelow EB. Papillary squamous cell carcinoma of the head and neck. Frequent infection with human papillomavirus infection and invasive carcinoma. Am J Surg Pathol. 2009;33:1720-4.

42. Mehrad M, Carpenter DH, Chernock RD, Wang H, Ma X-J, Luo $\mathrm{Y}$, et al. Papillary squamous cell carcinoma of the head and neck: Clinicopathologic and molecular features with special reference to human papillomavirus. Am J Surg Pathol. 2013;37:1349-1356.

43. Bishop JA, Westra WH. Human papillomavirus-related small cell carcinoma of the oropharynx. Am J Surg Pathol. 2011:35:1679-1684.
44. Kraft S, Faquin WC, Krane JF. HPV-associated neuroendoctine carcinoma of the oropharynx: a rare entity with potentially aggressive clinical behavior. Am J Surg Pathol. 2012;36:321-330.

45. Abeler VM, Holm R, Nesland JM, Kjørstad KE. Small cell carcinoma of the cervix. A clinicopathologic study of 26 patients. Cancer. 1994;73:672-7.

46. Wang KL, Yang YC, Wang TY, Chen JR, Chen TC, Chen HS, et al. Neuroendocrine carcinoma of the uterine cervix: A clinicopathologic retrospective study of 31 cases with prognostic implications. J Chemother. 2006;18:209-16.

47. Cai C, Chernock RD, Pittman ME, El-Mofty SK, Thorstad WL, Lewis JS. Keratinizing-Type Squamous Cell Carcinoma of the Oropharynx: p16 Overexpression Is Associated With Positive High-Risk HPV Status and Improved Survival. Am J Surg Pathol. 2014;38:809-15

48. Lu DW, El-Mofty SK, Wang H. Expression of p16, Rb and p53 proteins in squamous cell carcinoma of the anorectal region harboring human papillomavirus DNA. Modern Pathol. 2003;16:692-9.

49. El-Mofty SK, Lu DW. Prevalence of high-risk human papillomavirus DNA in nonkeratinizing (cylindrical cell) carcinoma of the sinonasal tract: a distinct clinicopathologic and molecular disease entity. Am J Surg Pathol. 2005;29:1367-72. 